\title{
policy statement
}

\author{
Prediction and Mitigation of Flash Floods \\ (Adopted by the AMS Council on 14 February 2000)
}

\section{Introduction}

In spite of decades of effort by government and the private sector to improve observations and warnings, flash floods continue to be one of nature's worst killers. For example, during an approximately 1 -h period on 5 May 1995, 16 lives were lost and over \$1 billion in property damage occurred as a result of a flash flood in Dallas, Texas. Similarly, on the evening of 28 July 1997, a flash flood in Fort Collins, Colorado, took five lives and caused over $\$ 100$ million in property damage. Events such as these ensure that flash floods, along with lightning, remain the leading weatherrelated causes of deaths in the United States.

Flash floods are distinguished from other types of flooding by the short timescales over which floodproducing rainfall occurs (generally less than $6 \mathrm{~h}$ ) and the small spatial scales (generally less than $1000 \mathrm{~km}^{2}$ ) of drainage basins in which flooding occurs. Most flash floods occur at night and, as would be expected, produce the most damage in urban areas. Aside from intense rainfall and small net storm motion, factors that contribute strongly to flash flooding are low permeability or saturated soils, impervious ground surfaces, and steep slopes. Failure of small to medium-sized dams, including debris dams, contributes significantly to the fatalities and damage associated with flash floods. A majority of flash-flood-related deaths occur in motor vehicles as people seek shelter and/or try to escape from rising waters.

Recreational activities and economic development continue to place pressures on floodplain utilization. As a result, the number of flood-prone communities grows larger and therefore the need for more timely and accurate warnings continues to increase. Although structural measures of protection (e.g., flood control reservoirs) can provide some protection from flooding from larger streams with longer flood lead times,

CC2000 American Meteorological Society it is too costly to use flood control structures on the large number of small streams flowing through populated areas. Moreover, it is difficult and costly to construct and then continuously monitor the safety of all structures that could mitigate flash flood disasters. Therefore, it is vitally important to reduce the population living in floodplains by providing disincentives to encroachment. This is one of the goals of the national flood insurance program of the Federal Emergency Management Agency (FEMA). Nevertheless, since too many people still dwell along small streams that can easily flood, community warning systems and self-help programs provide the only practical safeguard for many small flood plain communities.

\section{Warning systems and new technologies}

Essential components of flash flood warning systems include the following.

- Preparedness programs that include local citizen involvement.

- Rainfall-observing systems.

- Electronic data communication systems.

- Diagnostic/predictive models.

- Model calibration procedures.

- Warning dissemination systems.

- Action plans for local civil authorities.

In fact, many of the accomplishments in forecasting and improved warnings for flash flooding have come from the application and utilization of a number of these program components. For example, the National Weather Service (NWS) lead time for flash flood forecasts has increased to over 50 minutes. Much of this improvement is directly tied to new technology and also training along with the introduction of hydrometeorologists in the Weather Forecast Offices. The deployment of the new weather surveillance radars na- 
tionwide has significantly improved our capability to continuously monitor intense localized rainfall.

Furthermore, upgrading the new radar network to include polarimetric capabilities may offer substantial potential for significant additional improvements in rainfall measurements. The new radar technology, when combined with satellite and advanced automated surface rainfall and streamflow technologies, enhances the prospects for detecting and quantifying intense rainfall and rapid rises in streamflow. Still further, the databases created by the new observing capabilities facilitate better studies of the physical character of such rainfall events. This is especially true for understanding flash flood dynamics and microphysical processes, knowledge that is vital for the development of improved radar rainfall estimates. For example, improved understanding of radar underestimation of "warm-process" rain will ultimately lead to further improvements in diagnostic and forecast models. Because both remotely sensed and on-site recorded data are necessary for the production of high-resolution (to a few square kilometers) optimal estimates of rainfall, deployment and maintenance of rain gauge networks will still be important components of hydrologic research and development. A major challenge for remote sensing systems is enhancement of rainfall estimation capabilities in complex terrain.

\section{Integrated hydrometeorological approaches}

The coupled meteorological-hydrological nature of flash floods is becoming more and more evident. Prediction of flooding events will require interactive meteorological and hydrological models that introduce the new weather radar data and include feedbacks from the near-surface soil water to the atmosphere. Coupled hydrological-meteorological models should be constructed in a manner that permits prediction of the time and space distribution of both the rainfall and the resultant flooding. Efforts to predict the temporal and spatial distribution of flooding are expected to dominate research and development of diagnostic/predictive models. Considerable efforts have also been expended to develop probabilistic approaches to heavy rainfall and flood forecasting. Early efforts during the late 1990s have shown dramatic improvements in warnings when forecasters are trained and fully engaged in understanding the strengths and drawbacks of utilizing probabilities to express their confidence in their rainfall forecasts. Additional work also must be done to improve automated stream gauging systems and to improve integrated rainfall-streamflow prediction systems that integrate models with observations.

\section{Important research challenges include}

- understanding the processes that govern the production of extreme rainfall rates in convective weather systems,

- determining why some convective systems become quasi-stationary,

- defining the effects of the initial spatial distribution of soil moisture on the development of surface runoff,

- developing reliable distributed hydrologic models for simulating the hydrologic response of urban areas,

- linking local distributed hydrologic models to the larger-scale hydrologic models used operationally by the river forecast centers of the NWS,

- establishing the fundamentals of small-catchment hydrology/hydraulics, and

- quantifying forecast uncertainty by providing probabilistic forecast guidance.

The influence of the spatial scales of soil moisture on flash flood modeling is central to supporting these basic research efforts. Data requirements for conducting the research and developing predictive models necessitates the utilization of geographic information systems for specifying catchment geometrical properties pertinent to surface runoff development.

Because the occurrence of flash floods depends strongly upon the local nature and dynamics of rainfall and upon the wide spectrum of spatial and temporal scales that such dynamics span, fundamental uncertainties are inherent in any attempt to forecast these events. These uncertainties make it necessary to use statistical-dynamical predictive rainfall models. They also encourage exploration of probabilistic approaches, including ensemble forecasting techniques.

\section{Preparedness and self-help programs}

Dissemination of flood warnings has improved in recent years, largely in response to the enhanced coverage and attention of the local commercial media. Public response to warning would improve if individual streams were identified in the warning mes- 
sages. Also, enhanced National Oceanic and Atmospheric Administration Weather Radio coverage of flash flood-prone areas over the United States would undoubtedly contribute to smaller disaster impact. Dissemination methods involving the Internet should be explored and tested.

Proactive preparedness programs remain indispensable for loss-of-life and flood-damage reduction. Continued efforts by the NWS, FEMA, the media, and state and local emergency management agencies to educate the public regarding the occurrences and destructive force of flash floods are essential. Improved community monitoring, detection, and warning programs with emphasis on individual warning responses are a must. Real-time feedback from local designated persons or authorities to the National Weather Service Forecast Offices as to the hydrological and meteorological aspects of flood development will make for a more effective warning system.

\section{Summary}

Present-day flash floods are calamities with the potential for a very high death toll and huge losses of property. Although forecasting such events remains a tremendous challenge, the availability of the new weather radar data is expected to enhance forecast reliability, at least for short forecast lead times. Nevertheless, integrated hydrometeorological approaches based on sound science and new technological advances are necessary if more reliable and timely predictions are to become a reality. Coordinated dissemination and preparedness programs that involve individual and government initiatives will remain essential for effective flash-flood hazard mitigation.

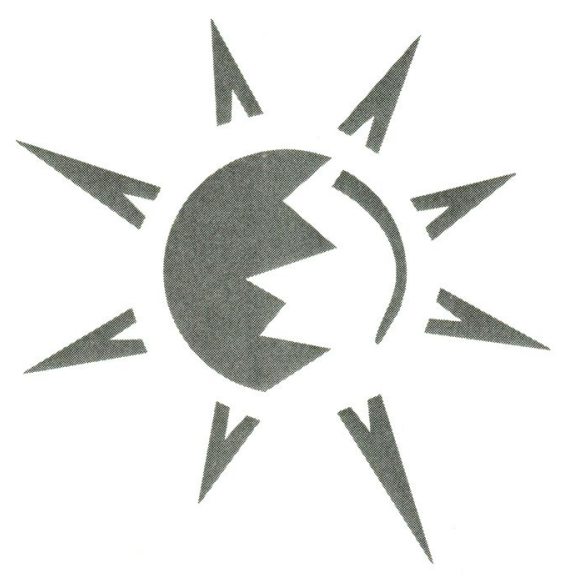




\title{
policy statement
}

\author{
Hurricane Research and Forecasting \\ (Adopted by AMS Council on 14 February 2000)
}

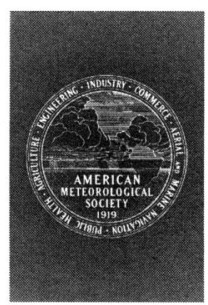

\section{Introduction}

Hurricanes ("typhoons" in the western North Pacific or "cyclones" in the Indian Ocean and throughout the Southern Hemisphere) belong to a class of phenomena referred to generically as tropical cyclones. The term hurricane is applied whenever the surface (33 ft or $10 \mathrm{~m}$ ) winds reach a one-minute sustained speed of $64 \mathrm{kt}\left(74 \mathrm{mph}\right.$ or $33 \mathrm{~m} \mathrm{~s}^{-1}$ ). Although there are some differences in strength and behavior of tropical cyclones in the various regions of the Tropics, their dynamics are similar. This statement examines the hurricane problem from a scientific and research point of view. A previous AMS policy statement (Bull. Amer. Meteor. Soc., 67, 537) addressed the hurricane warning system and preparedness efforts.

Our ability to understand, forecast, and respond effectively to hurricanes is tested every summer. The 1995-98 Atlantic hurricane seasons constituted the most active period of four consecutive years on record, which stretches back reliably at least to the advent of aircraft reconnaissance in the mid-1940s. As many of these hurricanes approached and battered North America, the importance and need for accurate forecasting of hurricane movement, intensity, and size and for improved emergency preparedness planning and response have been made clear to its residents.

\section{Scientific understanding of tropical cyclones}

Tropical cyclones form from frequently occurring groups of loosely organized, deep cumulus cloudstermed tropical disturbances - that occur in a variety of tropical weather situations. The most common pattern for storm genesis in the Atlantic is intensification of tropical easterly waves that regularly move off the west

C2000 American Meteorological Society coast of North Africa during the late summer and early autumn. The hurricane is a rare phenomenon; most tropical waves do not evolve into tropical cyclones.

The climatology of Atlantic tropical cyclogenesis suggests that formation is favored when a strong convective disturbance occurs in a region where the air is already spinning in a cyclonic (counterclockwise) direction. Other favorable factors include weak vertical wind shear, low-level inflow, and high-level outflow. Interactions between these incipient disturbances and upper-tropospheric troughs often contribute to cyclone development. Genesis almost always occurs over tropical waters with temperatures of at least $80^{\circ} \mathrm{F}$ $\left(26.5^{\circ} \mathrm{C}\right)$. The initiation of a vortex with tropical storm force-wind (34 kt or $39 \mathrm{mph}$ or $18 \mathrm{~m} \mathrm{~s}^{-1}$ ) can occur very rapidly, often in less than one day. The dynamics of the initial stage of the tropical cyclone are not well understood, owing to a comparative paucity of observations throughout the troposphere in the regions of storm genesis and the complexity of the interactions among the many scales of motion.

Intensification of a weak circulation into a hurricane can be thought of as the evolution of a nearly circular vortex in which the dominant forces are in approximate balance. The balance of forces near the sea surface is altered by friction, causing air to spiral inward toward the storm center. Clouds near the center become organized into spiral-rainband structures and, eventually, an eyewall by the strong rotations in the vortex. The strengthening winds and lowering surface pressure extract increasing amounts of water vapor from the warm ocean. As air rises and cools near the storm center, this water vapor condenses, releasing latent heat. The heating of the central region of the storm leads to its intensification, thus increasing the surface winds and evaporation, and so on. Unless impeded by an unfavorable environment, the storm will continue to intensify until the energy input by surface evaporation is balanced by frictional dissipation.

The reasons that some disturbances intensify to a hurricane, while others do not, are not well understood. 
Neither is it clear why some tropical cyclones come close to reaching their thermodynamic potential as major hurricanes [category 3, 4, or 5 on the SaffirSimpson hurricane scale with winds of at least $96 \mathrm{kt}$ (111 mph or $50 \mathrm{~m} \mathrm{~s}^{-1}$ )], while others do not. These major hurricanes produce $80 \%-90 \%$ of the U.S. hurricane-caused damages despite accounting for only one-fifth of all landfalling tropical cyclones.

Although small-scale details of the storm change continuously and sometimes rapidly, the tropical cyclone, as a whole, is a stable system that may persist for many days over sufficiently warm water. During this time, a tropical cyclone moves in the general direction of the broad-scale, deep layer average wind in which it is embedded. Tropical cyclones dissipate rapidly after landfall, owing primarily to the loss of the surface energy source. The vortex may retain some organization, particularly in the middle troposphere, for several days after landfall. Storms that move poleward over cold waters tend to weaken at a slower rate than those storms that move over land. Tropical cyclones that reach subtropical or midlatitude regions often interact with frontal weather systems and dissipate, owing perhaps to higher vertical wind shear. However, approximately half of these interactions lead to a transition of the tropical cyclone into an intense extratropical cyclone. The transformed cyclone has a different structure with weaker peak winds, but still has large areas of substantial rainfall and damaging winds.

\section{Hurricane-related hazards}

In the United States coastal zone, extensive damage and loss of life are caused by the storm surge (a rapid, local rise in sea level associated with storm landfall), heavy rains, strong winds, and hurricanespawned tornadoes. The continental United States currently averages nearly $\$ 5$ billion (in 1998 dollars) annually in tropical cyclone-caused damage, and this is increasing, owing to growing population and wealth in the vulnerable coastal zones.

Before 1970, large loss of life stemmed mostly from storm surges. The height of storm surges varies from 3 to $5 \mathrm{ft}(1-2 \mathrm{~m})$ in weak systems to more than $20 \mathrm{ft}(6 \mathrm{~m})$ in strong hurricanes that strike coastlines with shallow water offshore. The storm surge associated with Hurricane Andrew (1992) reached a height of about $17 \mathrm{ft}(5 \mathrm{~m})$, the highest level recorded in southeast Florida. Hurricane Hugo's (1989) surge reached a peak height of nearly $20 \mathrm{ft}(6 \mathrm{~m})$ about
20 miles northeast of Charleston, South Carolina, and exceeded $10 \mathrm{ft}(3 \mathrm{~m})$ over a length of nearly 100 miles of coastline. In recent decades, large loss of life due to storm surges in the United States has become less frequent because of improved forecasts, fast and reliable communications, timely evacuations, a better educated public, and a close working relationship between the National Hurricane Center (NHC), local weather forecast offices, emergency managers, and the media. Luck has also played a role, as there have been comparatively few landfalls of intense storms in populous regions in the last few decades. The rapid growth of coastal populations and the complexity of evacuation raises concerns that another large storm surge disaster might occur along the eastern or Gulf Coast shores of the United States.

In regions with effectively enforced building codes designed for hurricane conditions, wind damage is typically not so lethal as the storm surge, but it affects a much larger area and can lead to large economic loss. For instance, Hurricane Andrew's winds produced over \$25 billion in damage over southern Florida and Louisiana. Tornadoes, even though they occur in many hurricanes that strike the United States, generally account for only a small part of the total storm damage.

While hurricanes are most hazardous in coastal regions, the weakening, moisture-laden circulation can produce extensive, damaging floods hundreds of miles inland long after the winds have subsided. In recent decades, many more fatalities in North America have occurred from tropical cyclone-induced inland flash flooding than from the combination of storm surge and wind. For example, although the deaths from storm surge and wind along the Florida coast from Hurricane Agnes in 1972 were minimal, inland flash flooding caused more than 100 deaths over the northeastern United States. More recently, rains from Hurricane Mitch (1998) killed at least 10000 people in Central America.

Not all hurricane-related phenomena are detrimental. Hurricane-related rainfall, for example, has sometimes benefitted drought-stricken areas.

\section{The state of hurricane monitoring and forecasting}

\section{a. Hurricane monitoring}

As tropical cyclones usually form far from land and spend much of their life over remote oceanic areas, some storms escaped detection prior to the $1960 \mathrm{~s}$, particularly in those regions not subject to routine air- 
craft reconnaissance. Geostationary weather satellites have largely solved the detection problem and have improved the monitoring of these storms. However, satellite-derived position estimates may be in error by tens of miles and wind speed estimates may err by tens of knots. Although advances have been made using microwave imagery, it is still not possible to accurately or completely determine surface wind field distributions or detailed structural characteristics of tropical cyclones using satellite-based measurements alone. A combination of observing systems appears to be necessary for optimal analyses.

Supplementing weather satellite data are measurements from reconnaissance aircraft, coastal radars, ships, buoys, and land stations. Reconnaissance aircraft with special instrumentation can observe tropical cyclones within range of bases ashore. They provide accurate information on the storm's position as well as structure and intensity data. Airborne Doppler radar can capture the three-dimensional wind field from the surface to over seven miles in height. Similarly, active scatterometers and passive radiometers can sense surface winds in great detail from a flight altitude of several miles. Global positioning system-(GPS) based dropwindsondes deployed from aircraft provide detailed wind, temperature, and moisture information from flight level to the surface, even in the violent, inner core eyewall region. Despite advances in remote sensing capabilities from satellites, in situ measurements from reconnaissance aircraft remain essential for accurate monitoring of landfalling hurricanes.

\section{b. Hurricane track forecasting}

The tropical cyclone track forecast is a critical component of the warning system. Track prediction serves as the basis for forecasting the areas threatened by hurricane winds, rainfall, and storm surge. Normally, motion forecasts out to 3 days are issued every $6 \mathrm{~h}$, but demand for 5-day forecasts is emerging.

The local environmental atmospheric flow in which the tropical cyclone is embedded is the main factor that determines its short-term track. At longer timescales, an accurate forecast of the flow at considerable distance from the cyclone is needed. The internal structure of the tropical cyclone and interaction between this structure and the cyclone's environment are secondary influences on storm motion. Accurate track prediction requires detailed measurements on scales from the storm's large-scale environment to its small inner core.
Aircraft reconnaissance center fixes have been found to be necessary for accurate short-range ( 0 $24 \mathrm{~h}$ ) forecasts of hurricane landfall, wind field, and storm surge. Satellite-derived wind information has been increasing in coverage and accuracy, leading to improved large-scale analyses and model initial conditions. Additionally, to provide optimum determination of the full tropospheric flow around tropical cyclones, GPS dropwindsondes are dropped in a synoptic pattern around the storm from a National Oceanic and Atmospheric Administration (NOAA) Gulfstream IV jet and WP-3D aircraft. The use of these aircraft-deployed GPS dropwindsondes provides significant improvements in track forecasts made within a few days of landfall.

Dramatic improvement in the tropical cyclone track forecasts by dynamical models (such as that developed by NOAA's Geophysical Fluid Dynamics Laboratory) have made them the primary guidance for the forecaster as their accuracy exceeds that of statistical models previously used. Nonetheless, inconsistencies among guidance products place heavy demands upon the forecaster. As in other forecast problems, tropical cyclone prediction relies on a subjective mix of computer guidance and human interpretation and understanding, so that forecaster skill and experience are critical for success.

NOAA NHC track forecast errors - the distance between a forecast and the subsequently observed position of the storm center-for the decade 1989-98 averaged 47 (87), 87 (162), 162 (301), and 242 (449) $\mathrm{n} \mathrm{mi} \mathrm{(km)}$ for the Atlantic 12-, 24-, 48-, and 72-h forecasts, respectively. Using a combination of climatology and persistence as a basis for comparison, track forecast skill exists at all time intervals out to $72 \mathrm{~h}$, with the 48-h forecast showing the highest level of skill. Forecast errors have large spatial variations, averaging up to $30 \%$ greater than the mean in the central Atlantic and up to $30 \%$ less than the mean over the Gulf of Mexico and the Caribbean Sea. These differences arise from data availability as well as from the variable characteristics of hurricane motion.

During the four very active Atlantic hurricane seasons of 1995-98, NHC 24-h forecast errors were $20 \%$ lower than in the 1980s. This dramatic reduction may be attributed to various factors, including the use of improved data assimilation methods and better tropospheric data coverage from GPS dropwindsondes and satellite-based information, improvements in dynamical track forecasting models, and application of improved knowledge of the processes affecting storm motion. 
Given current forecast accuracy, it is necessary to issue hurricane warnings for large coastal areas. Warnings issued about $24 \mathrm{~h}$ before hurricane landfall cover an average of $400 \mathrm{n} \mathrm{mi}(740 \mathrm{~km})$ of coastline. Normally, the damage swath encompasses about onequarter of the warned area. Thus, approximately threequarters of the area is, in effect, "overwarned." Such overwarning is financially costly (estimates vary between $\$ 500,000$ to $\$ 1,000,000$ per coastal mile for hurricane warnings issued in the United States). There is conflicting evidence about the effect of this overwarning on societal response to future warnings. The total number of coastal miles warned has increased since the 1960s for several reasons, some nonmeteorological. For example, in response to increasing evacuation clearance times, hurricane warnings are now issued with greater lead time. Because uncertainties in hurricane forecasting increase with forecast period, this requires larger warning areas. Underwarning, of course, can lead to death and injury. The net effect on public safety, however, of all changes to the hurricane warning program during this era of rapid coastal population growth is that there have been less than ten storm surge deaths in the United States since 1970, compared to thousands in previous periods of comparable length. Hurricane warnings issued for U.S. coastal regions thus must carefully balance the need to minimize the economic burden of overwarning against the need to safeguard lives.

\section{c. Forecasting hurricane intensity and coastal hazards}

Numerical models can predict the storm surge inundation given a sufficiently accurate forecast of the hurricane's track and surface wind structure. However, because of current inaccuracies in track, intensity, and structure forecasting, overwarning of storm surge remains a problem.

Considerable improvement is needed in the understanding and prediction of tropical cyclone intensity change. On average, present operational forecasts are slightly better than those based on persistence and climatology. Mean NHC absolute errors of maximum hurricane wind speed for the period 1990-98 are 6.8 (3.5), 10.4 (5.3), 15.9 (8.2), and 19.7 (10.1) kt $\left(\mathrm{m} \mathrm{s}^{-1}\right)$ for the 12-, 24-, 48-, and 72-h forecasts, respectively. [Errors in estimating current storm intensity are considered to be in the neighborhood of $5 \mathrm{kts}\left(2-3 \mathrm{~m} \mathrm{~s}^{-1}\right)$.] These statistics do not portray the occasional large misses that typically occur when storms strengthen or weaken rapidly (as in the case of Hurricane Opal over the Gulf of Mexico in 1995).
Variations in hurricane size as well as transitions to extratropical storms can cause dramatic changes in storm impacts. The inability to anticipate these changes in intensity and structure is of great concern and a subject of intense research.

Skillful prediction of rainfall from landfalling tropical cyclones remains elusive. The prediction problem is complicated by terrain effects, the low inherent predictability of convective rainfall, and uncertainties in the track forecast. Evaluation of rainfall forecasts remains problematic, though there have been substantial improvements in precipitation estimates thanks to research aircraft and coastal Doppler radars. Satellite-based rainfall estimates, neural network statistical forecast models, and mesoscale numerical models appear promising for improvements in precipitation forecasts. But though objective guidance is steadily improving, rainfall prediction remains highly subjective.

\section{d. Forecast responsibilities of local and national weather offices}

Although the National Hurricane Center has overall responsibility for issuing track and intensity forecasts for Atlantic tropical cyclones, other national and local National Weather Service (NWS) offices play large roles as well in the United States. The forecasters at local National Weather Service Forecast Offices use their knowledge of the regional meteorology, oceanography, topography, and the population's demographics to provide specific information about the expected wind speed and direction, rainfall, storm surge, and hurricane-spawned tornadoes to local emergency management offices and the general public. In addition, the Hydrometeorological Prediction Center provides guidance on the potential for heavy rainfall both to the local NWS offices and directly to emergency management agencies, while guidance on the probability of severe local weather, such as tornadoes, is provided by the Storm Prediction Center.

\section{e. Seasonal hurricane forecasting}

Predictions of seasonal hurricane activity have demonstrated modest forecast skill since the mid1980s. These empirically based forecasts have been able to anticipate nearly $20 \%$ of the variance of hurricane frequencies when issued at the start of the season in early June. Such predictions, when based upon the phase of El Niño-Southern Oscillation, vertical wind shear, and Atlantic sea surface temperatures, among other factors, provide an overall guide to the 
activity expected during an upcoming Atlantic hurricane season. However, these forecasts have diminishing skill when issued several months before the beginning of the season. Improvements in seasonal forecasting may be possible with rigorous statistical techniques that can increase the skill, extend the lead time, and regionalize the predictions to specific locations. While seasonal hurricane forecasting is currently feasible only with statistical models, coupled global climate models could provide useful guidance in the future.

\section{Prospects and priorities for the next decade}

Despite large reductions in track forecast errors from dynamical models in recent years, operational hurricane forecast errors have not reached estimated predictability limits. While it is obviously desirable to continue to improve objective guidance through improved numerical models, data assimilation methods, and statistical techniques as well as better data, more needs to be done to quantify forecast uncertainty. Forecasters already rely on multiple numerical forecasts from several numerical weather prediction centers; more systematic development and implementation of ensemble techniques would likely have a large payoff both in improving the most probable track forecast and in quantifying its uncertainty.

In contrast to the improvements in track forecasts noted above, there has been little improvement in forecasts of storm intensity and surprisingly little effort to forecast and verify storm structure, including overall size. It is encouraging that the latest high-resolution hurricane prediction models provide improved representation of the hurricane inner core structure. These new models show promise for predicting trends in the storm intensity since they can resolve mesoscale features (eye, eyewall, spiral bands) important for intensity and structure changes. It has also become clear that accurate prediction of intensity, and perhaps structure, will depend crucially on coupling atmospheric models to ocean models. In this regard, the Geophysical Fluid Dynamics Laboratory (GFDL) hurricane prediction system, when coupled to an ocean model, has shown significant improvement in intensity predictions. But because of the low skill of present hurricane intensity forecasts, a need still exists for development and use of statistical and mixed dynamical-statistical intensity forecasting techniques. Indeed, the most skillful tool available to NHC forecasters is the Statistical Hurricane Intensity Prediction Scheme (SHIPS). Further research is needed to improve our quantitative understanding of the physical processes governing tropical cyclone intensity change.

Tropical cyclogenesis remains a vexing research issue. Although there have been notable advances in basic understanding, we appear to be some distance from accurate prediction of genesis. Global numerical weather prediction models do show some promising improvement in genesis forecasts, however.

Data assimilation for analysis and numerical forecasting of tropical cyclones presents a unique set of challenges. Many of the data assimilation techniques in use today were designed for circumstances in which the departures of the first-guess field from reality are small; while this appears to work well for most weather systems, it is clearly inadequate in the case of small-scale, intense coherent vortices such as hurricanes. Research into improved techniques for assimilating data when intense, coherent structures are present in the first-guess fields as well as the data might go a long way toward improved track prediction. At present, in the absence of in situ or remotely sensed observations, synthetic data can aid in the representation of the tropical cyclone vortex in the initial conditions. Improved data assimilation may also lead to improved analyses using existing but underutilized data sources. For example, advanced assimilation of satellite-based precipitation estimates may provide large improvements in the distribution of diabatic heat sources and tropical winds.

It is becoming apparent that the value of an observation in improving the analysis varies greatly from place to place and time to time; it has even been demonstrated that isolated observations at just the wrong place and time, even if free of error, can degrade an analysis. Development of advanced adaptive sampling techniques may be expected to lead to more effective measurement strategies, increasing the benefits and possibly reducing the costs of the measurements.

Numerical forecast models can and should be used to make estimates of the potential forecast value of new data sources, so that the costs and benefits of such new data sources can be assessed on a rational basis. For example, while GPS dropwindsondes deployed by the NOAA Gulfstream IV and WP-3D aircraft provide detailed information from the near-storm environment that improve forecasts by at least $20 \%$ through $48 \mathrm{~h}$, coverage is insufficient to improve longer range forecasts. Greater coverage may be achievable economi- 
cally with unmanned aircraft and by further use of wind information inferred from satellite data. Additional potential data sources include advanced satellite instrumentation, moored and deployed oceanic buoys, overthe-horizon radar, airborne remotely sensed data, and aircraft-deployed bathythermographs. Measurements of air-sea interaction, including sea surface temperature and upper-ocean heat content, are needed both for research and to support operational coupled oceanatmosphere hurricane models.

The same methods used to assess the potential value of new data sources can be used to estimate the effects of ongoing reductions in key elements of the observational network, such as rawinsondes, surface stations, and buoys.

The infrastructure in support of hurricane forecasting is undergoing important changes. Acquisition of high-performance supercomputers by NOAA has lagged considerably behind other nations, resulting in increasing use by the United States and other nations of models developed and run outside the United States. Partly as a response to this, it is likely that the private sector will undertake significant efforts in numerical weather prediction in the coming decade. As the private sector begins to generate and disseminate numerical weather predictions-based products, careful thought needs to be given to the terms of cooperation among the private sector, government, and universities to ensure that the public, private-sector interests, and emergency preparedness agencies receive the best possible guidance.

The primary goal of hurricane monitoring and forecasting is to prevent loss of life and to reduce vulnerability to winds, storm surges, and other hazards. Unfortunately, evacuation times for some communities now exceed what can reasonably be expected from present and projected forecast accuracy. Thus, a concerted scientific effort to improve forecasts should be combined with community development and preparedness programs to reduce evacuation times (OFCM 1996). The U.S. Weather Research Program for hurricane landfall (Marks et al. 1998; Elsberry and Marks 1999) promises improved forecasts of track, intensity, surface wind, and rainfall as well as research on decision making and the technology transfer necessary to convert advances in science and technology into products useful to society.

\section{References}

Elsberry, R. L., and F. D. Marks Jr., 1999: The hurricane landfall workshop summary. Bull. Amer. Meteor. Soc., 80, 683-685.

Marks, F. D., and Coauthors, 1998: Landfalling tropical cyclones: Forecast problems and associated research opportunities. Bull. Amer. Meteor. Soc., 79, 305-323.

OFCM, 1996: National Plan for Tropical Cyclone Research and Reconnaissance, Office of the Federal Coordinator for Meteorology, FCM-P25-1996, 159 pp.

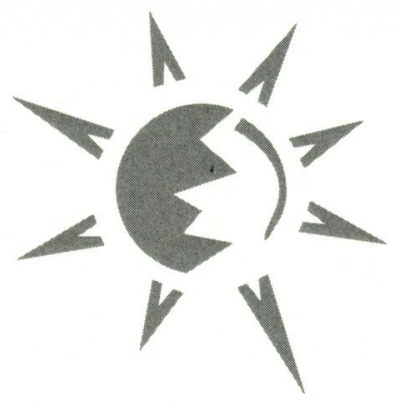




\section{policy statement}

\section{Guidance Statement on the Use of the Term "Live Radar" by the Broadcast Media (Adopted by AMS Council on 14 February 2000)}

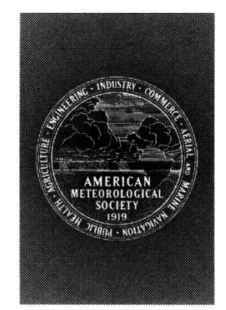

The broadcast media are a critical element of the public-private partnership, particularly in the dissemination of weather forecasts, watches, warnings, and advisories that enhance public safety and the protection of property (see Bull. Amer. Meteor. Soc., 80, 2099-2101). Major advances in radar technology and display over the past two decades allows for improved severe weather warning lead times, more accurate depiction of weather phenomena, and improved communication to the public.

The U. S. government's Next Generation Weather Radar ("NEXRAD" or "WSR-88D") network is composed of over 150 Doppler-capable units across the country, providing coverage for over $98 \%$ of the nation's population. It is a tri-agency program, managed by the National Oceanic and Atmospheric Administration/National Weather Service (NWS) on behalf of the U.S. Air Force and Federal Aviation Administration. Each radar independently scans the atmosphere, using a "volume scan," whereby every five to ten minutes, the radar interrogates the atmosphere at many levels. Dozens of graphical products are then generated that meteorologists can use to view weather phenomena. Through the public-private partnership, the NWS has an agreement (NEXRAD Information Dissemination System, or "NIDS") with a number of commercial organizations that receive these graphic products and make them available to end users such as the broadcast media in near-real time, in both unaltered and value-added forms. One of the most popular such value-added products is the radar mosaic, which combines the output of many single radars into one image, providing a wide-area view.

Many broadcast outlets have chosen to purchase and operate their own commercially available radar units, independent of the government. Some of these systems allow the operator to focus on local weather phenomena of interest and put the displays immediately on air. Although many of these radar units are not as sophisticated as NEXRAD, they do allow the media outlet to control the operation of the unit. Many media outlets choose to utilize both types of products on air, providing the advantages of each to their viewers. Because there may be uncertainty as to the timeliness of the radar products, the American Meteorological Society urges care in using the term "live radar" and recommends that all radar products be displayed with a "time stamp" that indicates the time the data in the image were acquired, and not the time that the graphic was generated, nor the time it is being displayed. In the case of severe weather, the public interest is best served in providing accurate, timely information and not hyperbole that might distract the viewer from the critical information necessary to protect lives and property.

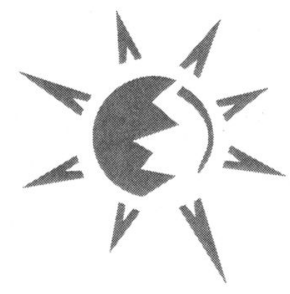

C2000 American Meteorological Society 